\title{
Superconducting tunnel junctions as detectors for ultraviolet, optical, and near infrared astronomy
}

\author{
T. Peacock, P. Verhoeve, N. Rando, M.A.C. Perryman, B.G. Taylor, and P. Jakobsen \\ Astrophysics Division, Space Science Department of ESA, ESTEC, Noordwijk 2200AG, The Netherlands
}

Received April 22; accepted September 27, 1996

\begin{abstract}
We discuss the capabilities of superconducting tunnel junctions as detectors for ultraviolet, optical, and near-infrared astronomy. Such junctions have recently been shown to allow the detection of individual optical and ultraviolet photons with an inherent spectral resolution related to the critical temperature of the absorbing superconductor. Limiting resolutions at $500 \mathrm{~nm}$ ranging from 5-40 nm (for materials with critical temperatures between 0.1 to $10 \mathrm{~K}$ ) should be achievable. These detectors should have a high quantum efficiency ( $>50$ per cent) over a very broad wavelength range from the ultraviolet to the near infrared $(100-2000 \mathrm{~nm})$. The overall efficiency is limited by reflection from the superconducting film, and should be improved significantly by appropriate anti-reflection coatings. The devices function at very high incident photon rates - with count rates of order $10 \mathrm{kHz}$ or higher being feasible, and photon arrival time datation possible to microsec-level accuracy. It is realistic in the future to envisage that these devices, of a size typically $20-50 \mu \mathrm{m}^{2}$, could be packaged into imaging arrays. These key characteristics imply that many areas of optical and ultraviolet astronomy could benefit significantly from their further development.
\end{abstract}

Key words: instrumentation: detectors instrumentation: spectrographs — ultraviolet: general

\section{Introduction}

The ideal detector for application in a variety of astronomical studies at ultraviolet, optical, and infrared wavelengths is one which can simultaneously over a wide waveband provide the wavelength, time of arrival and location of each photon falling upon the detector from the widest possible field. Intrinsic energy discrimination, although typical of modern X-ray detectors such as X-ray CCDs, does not occur in any existing ultraviolet or optical detector systems. Any detector development which provides these key features will make a major contribution to astrophysical research by improving the sensitivity limits for faint object spectroscopy, and by simultaneously providing the photon arrival time.

Perryman et al. (1993) proposed, largely on theoretical grounds, that optical detectors based on superconductors could make a major advance in the development of instrumentation for application in astronomy. Since this proposal experimental demonstration of the basic principles using niobium-based superconductors by Peacock et al. (1996a,b) has shown the validity of this approach. In this paper we extend these initial ideas to the more general case of an arbitrary superconductor, consider the effects of multiple tunnelling, and indicate the wavelength resolution that may ultimately be expected under various conditions.

Despite the considerable strengths which have made the CCD the pre-eminent detector for optical astronomy, three performance weaknesses still exist: the lack of an inherent spectral resolution, the limited quantum efficiency at short wavelengths (in the blue/ultraviolet), and the inability to photon count - and thereby provide the arrival time of the incident photon (Delamere 1992). Current photon counting detectors cannot compete with CCDs except with respect to time resolution, and for applications involving very low signal levels. None provides a measure of the photon wavelength, all have a limited quantum efficiency due to the available photocathode materials, and all are limited by their photon count rates (Timothy 1988; Petroff \& Stapelbroeck 1989). Photon-counting ability has potential applications in many fields of astronomy, e.g. the study of the optical properties of variable sources, as well as compensation for the effects of atmospheric seeing. It is however the lack of any intrinsic wavelength discrimination which is a serious limitation of all available photon detectors, and which the superconducting detector overcomes for the first time.

Detectors based on semiconductors have a bandgap (1.1 eV for silicon) of order of the (optical) photon energy. Photoabsorption results in typically only one electron being excited from the valence to the conduction 
band, irrespective of photon wavelength. This precludes, without some form of amplification, the possibilities of photon counting, and/or wavelength discrimination.

This is not however the case for superconductors. As broad-band, noiseless, high speed photon counting detectors, with inherent spectroscopic ability - and thus the ability to cover efficiently in a single detector the waveband from the ultraviolet to the near infrared with medium spectroscopic resolution - they could allow developments in a number of different fields of astronomy. For example the observation of emission-line complexes or continuum absorption features such as the Lyman edge in very faint extragalactic objects may allow the direct determination of their red shifts. Applications requiring time resolution, either to measure intrinsic variability characteristics (e.g. Dravins 1994) or to overcome atmospheric effects, will also become more accessible.

\section{The photoabsorption process in superconductors}

As identified by Perryman, et al. (1993) optical detectors based on superconducting materials offer two important advantages over those based on semiconductors: (a) according to the BCS theory of superconductivity the energy gap $\Delta$ between the ground state, as represented by the bound Cooper pairs, and the first excited state, containing the broken Cooper pairs known as "quasiparticles", is generally more than $10^{3}$ times smaller than the energy gap between the valence and conduction bands of a semiconductor (Bardeen et al. 1957); (b) the Debye energy $\Omega_{\mathrm{D}}$ is much larger than the superconducting energy gap, thereby allowing phonons created as a result of the photoabsorption process to break Cooper pairs and create free charge (Wood \& White 1969). For example in bulk Al $\Delta \sim 170 \mu \mathrm{eV}$ while $\Omega_{\mathrm{D}}$ is as large as $\sim 37 \mathrm{MeV}$.

In a superconductor the conduction electrons at a particular transition temperature interact with the lattice (an attractive electron-phonon interaction) which overcomes their mutual Coulomb repulsion leading to the formation of electron pairs. It is these Cooper pairs which carry the electrical current. The temperature at which the phase transition occurs, when electrons begin to form into condensates of pairs, is known as the critical temperature $T_{\mathrm{c}}$. The Debye energy can be interpreted here as the maximum energy associated with the vibrational modes of the lattice. Table 1 summarises some of the key parameters of some elemental superconductors.

At sufficiently low temperature (typically about an order of magnitude lower than the superconductor's critical temperature $T_{\mathrm{c}}$ ) the initial number of quasiparticles $N_{0}$ created as a result of the absorption of a photon of wavelength $\lambda$, can be in excess of any thermally induced population and is inversely proportional to the photon wavelength $\lambda$. In general, $N_{0}$ can be written:

$N_{0}(\lambda) \sim 710^{5} / \lambda \Delta(T)$
Table 1. Basic parameters of some elemental superconductors. $T_{\mathrm{c}}$ is the critical temperature, $\Omega_{\mathrm{D}}$ the Debye energy, and $\Delta$ the energy gap. $H_{\mathrm{c}}$ is the critical magnetic field above which the superconducting state of the material is either destroyed or modified

\begin{tabular}{lcccr}
\hline Material & $\begin{array}{c}T_{\mathrm{c}} \\
(\mathrm{K})\end{array}$ & $\begin{array}{c}\Omega_{\mathrm{D}} \\
(\mathrm{MeV})\end{array}$ & $\begin{array}{c}\Delta \\
(\mathrm{MeV})\end{array}$ & $\begin{array}{r}H_{\mathrm{c}} \\
(\mathrm{G})\end{array}$ \\
\hline Niobium & 9.20 & 23.7 & 1.550 & 1980 \\
Vanadium & 5.30 & 32.7 & 0.800 & 1420 \\
Tantalum & 4.48 & 20.7 & 0.664 & 830 \\
Aluminium & 1.14 & 36.9 & 0.172 & 105 \\
Molybdenum & 0.92 & 38.8 & 0.139 & 95 \\
Cadmium & 0.56 & 18.0 & 0.083 & 30 \\
Titanium & 0.39 & 36.2 & 0.059 & 100 \\
Hafnium & 0.13 & 21.7 & 0.020 & 13 \\
\hline
\end{tabular}

where $\Delta(T)$ is the temperature-dependent energy gap. The mean energy, $\epsilon$, required to create a single quasiparticle in $\mathrm{Nb}$ and $\mathrm{Sn}$ has been calculated to be $\sim 1.75 \Delta$ and $\sim 1.7 \Delta$ respectively (Kurakado 1982; Rando et al. 1992). The variance on $N_{0}(\lambda)$ defines the fundamental limit to the intrinsic resolution $d \lambda_{\mathrm{F}}$ of the superconductor. This limiting resolution, known as the Fano-limit, can be written as:

$d \lambda_{\mathrm{F}} \sim 2.810^{-3} \lambda^{3 / 2}[F \Delta(T)]^{1 / 2}$

where, in both equations, the bandgap $\Delta$ is in $\mathrm{MeV}$ and $\lambda$ is in nm. $F$ is the Fano factor (Fano 1947) which has been shown to be $\sim 0.22$ and $\sim 0.19$ for $\mathrm{Nb}$ and $\mathrm{Sn}$ respectively (Kurakado 1982; Rando et al. 1992). The values $\epsilon \sim 1.75 \Delta$ and $F \sim 0.2$ can be considered as typical of many of the elemental superconductors.

In superconductors such as $\mathrm{Nb}$ or $\mathrm{Al}$ operating at $\sim 0.1 T_{\mathrm{c}}$, with critical temperatures of 9.2 and $1.14 \mathrm{~K}$ respectively, the initial charge $N_{0}(\lambda)$ created by the photoabsorption of an optical photon with $\lambda=500 \mathrm{~nm}$ is of order $10^{3}$ and $10^{4}$ quasiparticles respectively. The corresponding Fano-limited wavelength resolution in $\mathrm{Nb}$ or $\mathrm{Al}$ is $\sim 17 \mathrm{~nm}$ and $\sim 4 \mathrm{~nm}$ respectively.

Equations (1) and (2) can be further generalised to any superconductor through use of the approximate BCS relation in the weak coupling limit which links the bandgap at absolute zero to its $T_{\mathrm{c}}$ such that $\Delta \sim 1.75 k T_{\mathrm{c}}$, where $k$ is Boltzmann's constant (for a strongly-coupled superconductor such as $\mathrm{Nb}$, a somewhat better approximation is $\Delta \sim 1.93 k T_{\mathrm{c}}$ ) giving:

$$
\begin{gathered}
N_{0}(\lambda) \sim 4.610^{6} \lambda^{-1} T_{\mathrm{c}}^{-1} \\
d \lambda_{\mathrm{F}}(\mathrm{nm}) \sim 510^{-4} \lambda^{3 / 2} T_{\mathrm{c}}^{1 / 2} .
\end{gathered}
$$

Figure 1 illustrates this fundamental limiting spectral resolution $d \lambda_{\mathrm{F}}$ for a number of elemental superconductors. For $\mathrm{Al}$ the resolution between Lyman- $\alpha(\lambda=121.6 \mathrm{~nm})$ and $\lambda \sim 2 \mu \mathrm{m}$ ranges from $\sim 0.7$ to $50 \mathrm{~nm}$ respectively, 
with $N_{0}(\lambda)$ ranging from $\sim 310^{4}$ to $\sim 210^{3}$. If a low $T_{\mathrm{c}}$ superconductor such as Hf were used, the Fano-limited resolution would, over the same wavelength band, range from 0.2 to $15 \mathrm{~nm}$.

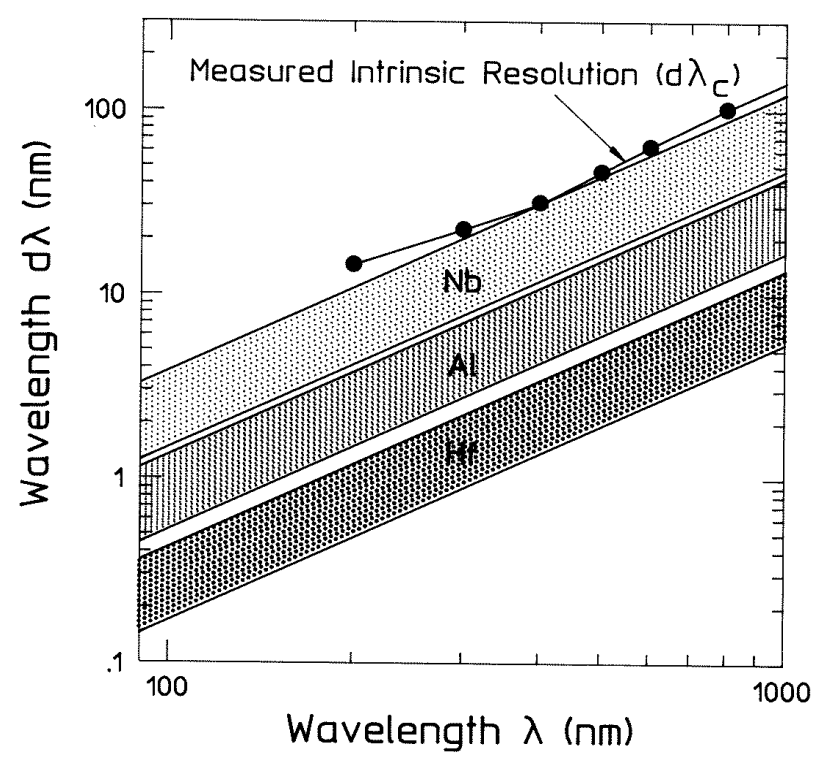

Fig. 1. The Fano and tunnel noise-limited resolution, $d \lambda_{\mathrm{F}}$ and $d \lambda_{\mathrm{T}}$ (lower and upper sloping lines respectively), versus wavelength for three superconducting materials. The electronics-corrected measured resolution $d \lambda_{\mathrm{c}}(\mathrm{nm})$ for a Nb-based symmetrical junction is also shown. The shaded regions indicate the range of possible resolutions $d \lambda_{\mathrm{F}}$ to $d \lambda_{\mathrm{T}}$ which will depend on the design of the STJ and the role of multiple tunnelling (Sect. 3.2)

\section{The superconducting tunnel junction}

\subsection{Principles of operation}

One of a number of ways of detecting the excess quasiparticles produced as a result of the photoabsorption process is by ensuring that they tunnel from one thin superconducting film $\left(S_{1}\right)$ in which they are created into another $\left(S_{2}\right)$ through a thin insulator $(I)$. To maximise the tunnel probability this insulating barrier must be very thin, of order $1 \mathrm{~nm}$ (i.e. only a few atomic layers). This superconductor-insulator-superconductor (or SIS) structure is in essence a superconducting tunnel junction (STJ) or Josephson junction (Giaever 1960; Josephson 1962). A small magnetic field is applied parallel to the junction barrier, as shown in Fig. 2, so as to suppress the Josephson current which results from the tunnelling of Cooper pairs at zero bias voltage. Applying a bias voltage $V_{\mathrm{b}}<2 \Delta / e$ ensures that the only allowed tunnel processes involve the transfer of quasiparticles from one film to the other. The flow of quasiparticles in a time $t$ produces a measurable excess electric current, with the amplitude of the current pulse being directly proportional to the incident photon energy.

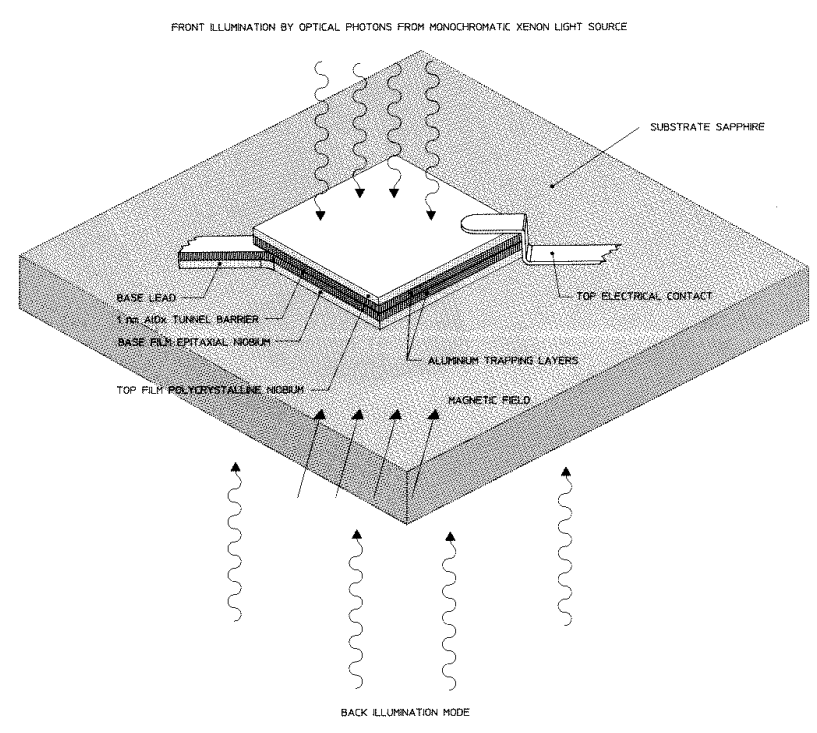

Fig. 2. A schematic of a typical symmetrical tunnel junction deposited on a sapphire substrate together with its orientation in a parallel magnetic field so as to suppress the Josephson current. Such a device was used by Peacock et al. (1996 a,b) to demonstrate single photon counting from $200-550 \mathrm{~nm}$. Back or front illumination is possible

\subsection{Multiple tunnelling and tunnel noise}

For the case where $V_{\mathrm{b}}<2 \Delta / e$ two tunnel processes exist (Gray 1978). In the first process an electron tunnels from film $S_{1}$ to film $S_{2}$ (the direction depends simply on the polarity of $V_{\mathrm{b}}$ ) and effectively a quasiparticle is exchanged, while in the second process an electron tunnels again from film $S_{1}$ to $S_{2}$ while a quasiparticle is exchanged between films $S_{2}$ and $S_{1}$. The combination of these two processes in series leads to an effect known as multiple tunnelling, which can be viewed as equivalent to an amplification of the initial charge $Q_{0}^{i}=e N_{0}^{i}(\lambda)$ created in the superconducting film $i$. If each quasiparticle originally created in the film is transferred across the barrier on average of $n$ times then, in the absence of loss processes, the average total electrical charge detected would be $n Q_{0}^{i}$. In this time-dependent process, recombination, diffusion losses and quasiparticle trapping will all reduce $Q_{0}^{i}(t)$ (Booth 1987; Verhoeve et al. 1996).

This charge amplification will however degrade the Fano-limited resolution of the STJ by adding in quadrature the variance on this tunnel process, a contribution referred to as the tunnel noise (Goldie et al. 1994). On the assumption of a perfectly symmetrical junction (with 
equal probabilities of tunnelling from $S_{1}$ to $S_{2}$ and from $S_{2}$ to $S_{1}$ ) the resolution is given by:

$$
d \lambda_{\mathrm{T}}(\mathrm{nm}) \sim 1.110^{-3} \lambda^{3 / 2} T_{\mathrm{c}}^{1 / 2}\left[F+\frac{n+1}{n}\right]^{1 / 2}, \quad n \geq 2 .
$$

This limiting resolution for a perfectly symmetrical STJ is also shown in Fig. 1 for the case $n=5$. This choice of $n$ is based on the experimental determination by Peacock et al. (1996a) for a symmetrical Nb-based device. Figure 1 shows that a resolution of $\sim 40 \mathrm{~nm}$ and $\sim 14 \mathrm{~nm}$ is achievable for simple $\mathrm{Nb}$ and $\mathrm{Al}$ based devices respectively when illuminated by photons of wavelength $\lambda \sim 500 \mathrm{~nm}$. The degradation due to an equivalent amplification through multiple tunnelling is not a basic limitation, since the need for such amplification is dependent on the signal-to-noise ratio and hence on the noise of the readout electronics. Provided the initial charge is sufficiently large compared to the electronic noise then such an amplification is not essential, and the contribution of the tunnel noise to the overall resolution can be reduced. Naturally, this requirement affects the basic design of the STJ and, depending on the materials used, its operating temperature. For these reasons we show the limiting resolution in Fig. 1 as a band ranging from $d \lambda_{\mathrm{F}}$ to $d \lambda_{\mathrm{T}}$, for the case $n \sim 5$.

Effects of the quasiparticle lifetime and the role of various recombination mechanisms were considered by Perryman, et al. (1993). For a typical $20 \times 20 \mu \mathrm{m}^{2}$ device with a film thickness of $100 \mathrm{~nm}$, i.e. a film volume $V \sim 40 \mu \mathrm{m}^{3}$, the number of thermal quasiparticles can be written:

$N_{\mathrm{T}} \sim 2 N(0) V(2 \pi \Delta k T)^{1 / 2} \mathrm{e}^{-\Delta / k T}$

where $N(0)$ is the single spin electronic density of states at the Fermi energy. From Eq. (3) a photon of wavelength $500 \mathrm{~nm}$ will produce an initial number of quasiparticles $N_{0}$ of order $10^{3}, 10^{4}$ and $10^{5}$ for $\mathrm{Nb}, \mathrm{Al}$ and $\mathrm{Hf}$ respectively. Combining Eqs. (3) and (6):

$N_{0} / N_{\mathrm{T}}=1.410^{10} T_{\mathrm{c}}^{-3 / 2}(\lambda N(0) V)^{-1} T^{-1 / 2} \mathrm{e}^{1.75 T / T_{\mathrm{c}}}$

It is reasonable to require that the thermal population is at least an order of magnitude lower than $N_{0}(\lambda)$. This means that, in the absence of additional barrier leakage mechanisms (thermal regime), the operating temperature $T$ has to be less than $\sim 0.1 T_{\mathrm{c}}, 0.15 T_{\mathrm{c}}$ and $0.24 T_{\mathrm{c}}$ for $\mathrm{Nb}$, $\mathrm{Al}$ and $\mathrm{Hf}$ respectively (i.e. $\sim 900,170$ or $30 \mathrm{mK}$ ) to detect $500 \mathrm{~nm}$ photons. In the event of multiple tunnelling, these requirements may be somewhat modified.

\section{Photon detection at optical wavelengths}

The superconducting tunnel junction has been extensively investigated as a photon detector in the X-ray region of the spectrum where the number of quasiparticles generated after photoabsorption are $\sim 10^{3}$ times larger than in the optical.
Peacock et al. (1996a,b) have recently reported the first experimental demonstration of photon counting at optical and ultraviolet wavelengths using an STJ. Single photon spectra from monochromated ultraviolet and optical photons covering the waveband $200-500 \mathrm{~nm}$ were obtained by illuminating a $20 \times 20 \mu \mathrm{m}^{2}$ device. This symmetrical device, which was constructed of aluminium-proximised niobium layers $[\mathrm{Nb}-\mathrm{Al}](\Delta \sim 0.5 \mathrm{MeV})$ operated at a temperature of $0.4 \mathrm{~K}$, is shown schematically in Fig. 2 . After removal of the contribution to the resolution from electronic noise a device-limited resolution $d \lambda_{c}$ was derived. Spatial variations in the response of the detector, similar to those reported by Verhoeve et al. (1996) and Lumb et al. (1995) at X-ray wavelengths, may account for the small degradation in resolution compared with that expected theoretically.

Recent performance improvements have been brought about by reducing the noise associated with the electronics, and by back illumination through the sapphire substrate onto the high quality epitaxial $\mathrm{Nb}$ film (cf. Fig. 2), leading to a wavelength coverage extended to $200-1000 \mathrm{~nm}$ (the short wavelength limit is simply limited by the experimental conditions). The device-limited resolution $d \lambda_{\mathrm{c}}$ is shown in Fig. 1 for this Nb-based device. A resolution $d \lambda_{\mathrm{c}}$ of $45 \mathrm{~nm}$ at $\sim 500 \mathrm{~nm}$ was obtained, very close to the theoretical value $d \lambda_{\mathrm{T}}$ for Nb shown in Fig. 1.

Figure 3 illustrates the single photon charge spectra, $Q$, obtained from this $\mathrm{Nb}$ device when illuminated with photons having a wavelength of 250 and $1000 \mathrm{~nm}$.

\section{Performance capability}

\subsection{Absorption efficiency and reflectivity}

The STJ has a very high efficiency to the absorption of photons of wavelength ranging from the ultraviolet to the near infrared. Figure 4a illustrates the absorption efficiency for single photons in $\mathrm{Nb}, \mathrm{Al}$ and $\mathrm{Hf}$ films of thickness $100 \mathrm{~nm}$ based on the optical constants of the materials (Weaver \& Lynch 1973; Weaver et al. 1981) and indirectly confirmed for Nb-based devices through optical transmission measurements in our laboratory. From $100 \mathrm{~nm}$ to $2 \mu \mathrm{m}$ the absorption efficiency is over 80 per cent for all three materials. The surface reflectivity of these films is shown in Fig. 4b. While pure $\mathrm{Al}$ has a very high reflectivity from the ultraviolet to the near infrared, typically over 90 per cent, the other two superconductors in Fig. 4b have a much improved capability. Hf in particular has a reflectivity below 30 per cent in the ultraviolet and maintains a value below 55 per cent out to $2 \mu \mathrm{m}$. For Nb, where an optically-sensitive STJ now exists, the reflectivity is below 50 per cent out to $700 \mathrm{~nm}$. The reflectivity, which affects not only the superconducting thin film but, depending on the mode of illumination (front illumination directly onto the top polycrystalline superconducting film or back illumination through the substrate onto the 

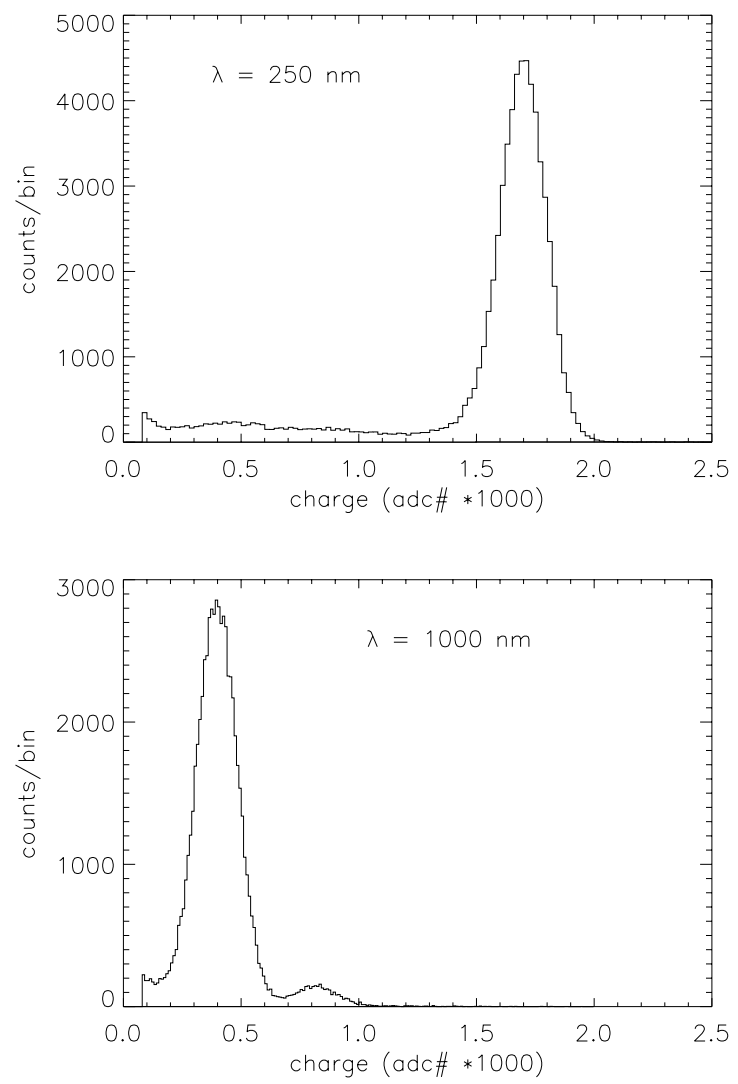

Fig. 3. Single photon charge spectrum of the device (a histogram of the number of photons giving a charge output $Q$ from the detector) when illuminated by a monochromatic source of photons of wavelength $250 \mathrm{~nm}$ (top) and $1000 \mathrm{~nm}$ (bottom). The charge is in arbitrary units. The difference in the charge output versus wavelength provides the intrinsic energy resolution.

epitaxial base film), also the substrate, and substrate to base film interface, can be reduced through the use of antireflection coatings, albeit at the expense of bandwidth and with device fabrication constraints. Such coatings are currently under development.

Further reduction in the electronic noise should lead to resolutions approaching the theoretical limits shown in Fig. 1. The resulting spectroscopic performance is illustrated in Fig. $5(N \sim 5)$ applied to emission line spectra from neutral (I) to quadruply ionised (V) atoms of oxygen and carbon. The line strengths (not corrected for abundances) and wavelengths are taken from Weast (1981). For the ion species III-V the line strengths should be considered as qualitative estimates of the relative strengths between different lines from the same atom. The intensity of the ionised atoms (II-V) relative to the neutral atom evidently depends on the excitation conditions. Figure 5 demonstrates that even the $\mathrm{Nb}$ device has a significant spectroscopic capability with many of the key lines of astrophysical interest being clearly resolvable.
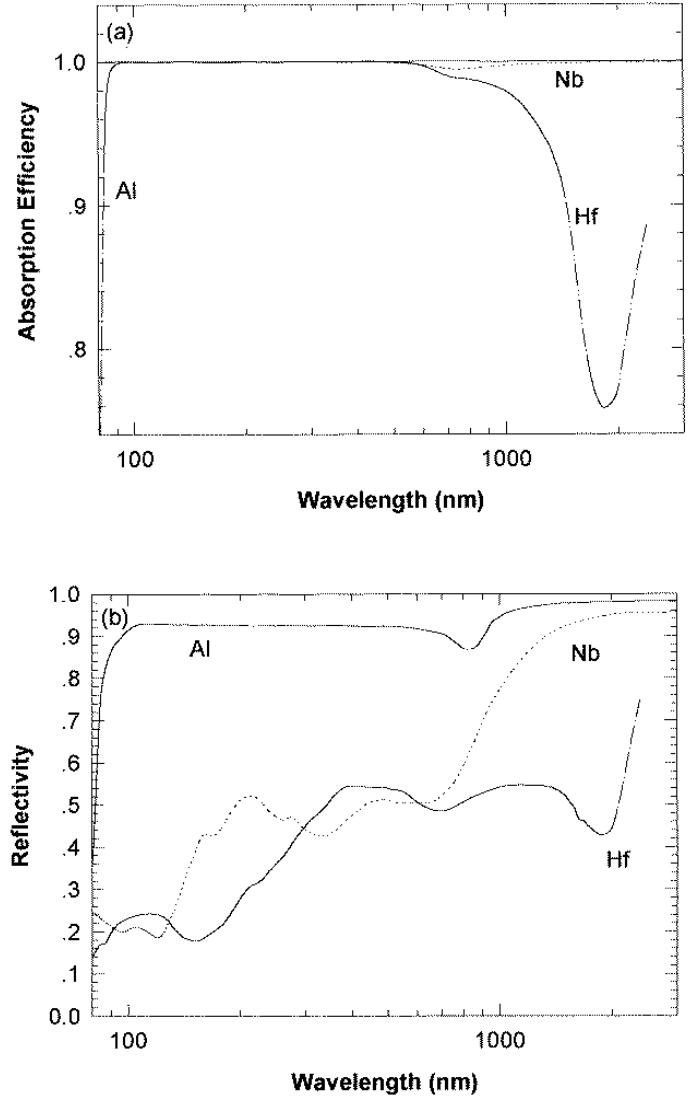

Fig. 4. The theoretical absorption efficiency, $A(\lambda)$ a) and surface reflectivity $R(\lambda) \mathbf{b}$ ) in a $100 \mathrm{~nm}$ film as a function of photon wavelength for $\mathrm{Nb}, \mathrm{Al}$ and $\mathrm{Hf}$. The reflectivity can be further decreased through the use of an anti-reflection coating at the expense of reducing the wavelength coverage. The overall quantum efficiency of the device for the case of front illumination is $A(\lambda) \times(1-R(\lambda))$.

The reflectivity of $\mathrm{Al}$ junctions is so high that the overall efficiency would limit considerably its usefulness at these wavelengths. While no devices based solely on $\mathrm{Hf}$ yet exist, it has formed the basis for some tunnel junction work (Morohashi et al. 1992). Nb- and Ta-based devices are however more standard, requiring only some improvements to achieve the theoretical limited resolutions of Fig. 1. From Fig. 5 however it is clear that an excellent spectroscopic capability would exist for devices based on Hf, with key transitions from the same ion as well as different ionic species being possible to resolve and identify.

\subsection{Count rates}

Another significant strength of superconducting tunnel junctions is the inherent speed of the device. The time taken to complete the full conversion of the photon energy into quasiparticles, including their relaxation down to an energy equal to the bandgap of the absorbing material, is only a few ns. The quasiparticle confinement time in the 

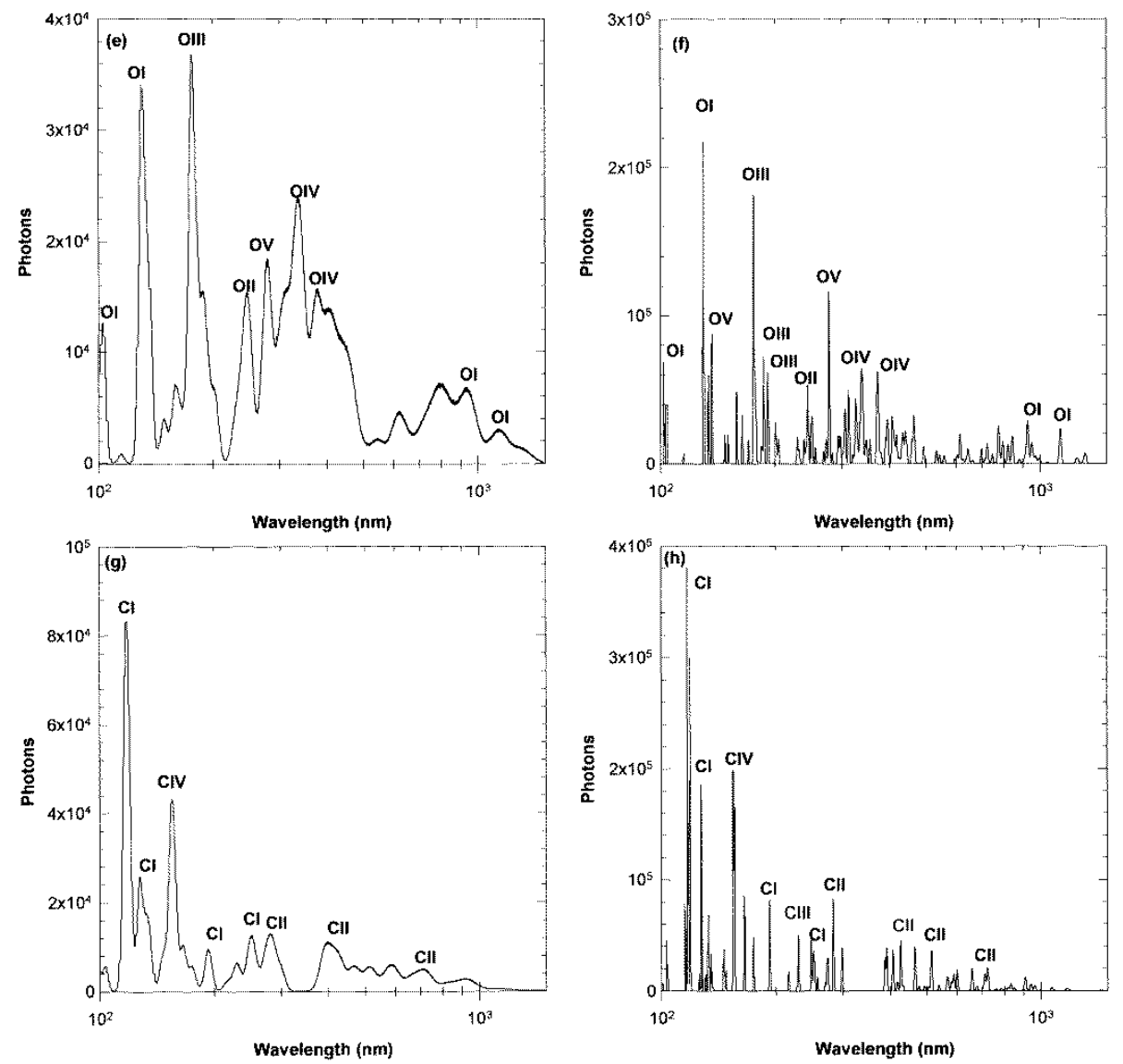

Fig. 5. The simulated tunnel-limited response $d \lambda_{\mathrm{T}}(n \sim 5)$ from either a pure Nb based STJ (left two diagrams) or Hf based STJ (right two diagrams) to the principle emission lines from oxygen (top pair) and carbon (bottom pair). The strongest lines from neutral and ionised species are marked. Note the photon scale is different in each case to highlight the various line features. For all these spectra the bin size was set at $0.1 \mathrm{~nm}$. No abundances are included in these simulations

film is complex and depends also on the superconducting material properties, bias voltage and film thickness. Based on the work of de Korte (1992) the confinement time in a single film can be written:

$\tau_{\text {tun }, i}=4 e^{2} N(0) V_{i} R_{\mathrm{N}} \frac{\left[\left(\Delta_{i}+e V_{\mathrm{b}}\right)^{2}-\Delta_{i}^{2}\right]^{1 / 2}}{\left(\Delta_{i}+e V_{\mathrm{b}}\right)}$

where $N(0)$ is the single spin electronic density of states at the Fermi energy, $e$ the electron charge, $R_{\mathrm{N}}$ is the normal state barrier resistance, $V_{i}$ is the volume of the film $i$ and $V_{\mathrm{b}}$ is the bias voltage applied across the two films. Thus for a device designed to provide a single tunnel from a $100 \mathrm{~nm}$ thick film of $\mathrm{Nb}$ across a barrier having a typical resistivity $\rho \sim 2.510^{-6} \Omega \mathrm{cm}^{2}$, the average confinement time is of order $100 \mathrm{~ns}$, scaling with the mean number of transfers $n$ across the barrier. The parameter $n \tau$ therefore represents approximately the intrinsic time resolution of the device.

Thus, in theory, superconducting tunnel junctions could be operated as photon counting detectors at rates of order $10^{5}$ to $10^{6} \mathrm{~Hz}$ depending on the device geometry and $n$. In practice Lumb et al. (1995) have shown rates as high as $\sim 7.5 \mathrm{kHz}$ can be achieved without any intrinsic spectroscopic degradation in the device, being limited by the processing speed of the analogue electronics and substrate noise associated with the X-ray photon energy used in the experiment. The electronics however will be the final limitation to the very important photon count rate capability at optical wavelengths. These rates will still allow very high speed photometry on objects to be limited primarily by the collecting aperture of the telescope as well as the observation of fields containing a objects having a wide dynamic range in intensity.

Since each photon results in an electrical pulse whose amplitude is directly proportional to the photon energy, and a pulse risetime which is characteristic of a single photoabsorption event in the film, the device has intrinsic background rejection capabilities. Background from cosmic rays can be vetoed either by an upper energy threshold discriminator or through the risetime signature.

For a panoramic imaging STJ camera covering a wide field of view a number of approaches can be considered. It must however be noted that each STJ detector is independent and requires its own analogue electronics chain 
including the preamplifier. This is a considerable limitation, the solution to which still needs to be fully addressed. Nevertheless, simple limited close-packed arrays of $6 \times 6$ STJs are already under development by our group (Rando et al. 1996), while Perryman et al. (1993) already proposed more complex schemes in an attempt to reduce the total number of detectors and associated electronic chains while maintaining field coverage.

Although the count rates that can be handled in photon counting mode are already expected to be very high, there may be applications (for example, at longer wavelengths, for very bright objects, or for large telescope apertures) where even these limits are exceeded. Under these conditions, it is likely that the relevant junctions can be read out in an analogue mode, in which the steady-state current is measured, rather than the pulse corresponding to an individual photon. In these circumstances, the STJ would simply provide the total energy incident on the junction, integrated over wavelength, with information on the energy per photon lost.

\section{Conclusion}

We have shown that superconducting tunnel junctions based on niobium or hafnium may have considerable potential as photon counting optical and ultraviolet detectors. The basic experimental feasibility has now been demonstrated, and the key features of such a detector can be summarised as follows: (a) photon counting operation with no readout noise, and with minimal dark current contribution; (b) an inherent spectroscopic capability at ultraviolet, optical, and infrared wavelengths, with tunnel noise-limited resolutions ranging from $0.4-4 \mathrm{~nm}$ at $100 \mathrm{~nm}$ and $5-50 \mathrm{~nm}$ at $500 \mathrm{~nm}$ for materials with $T_{\mathrm{c}}$ from $0.1-10 \mathrm{~K}$; (c) high quantum efficiency of 50 per cent or more over the range $100-2000 \mathrm{~nm}$. A higher efficiency over a more restricted waveband could be achieved through the introduction of appropriate anti-reflection coatings; (d) high speed, limited primarily by the processing electronics, leading to a high count-rate capability, and high resolution time datation (sub-ms) of individual photon events; (e) inherent discrimination against background events through the application of energy and risetime discrimination.

An illustrative list of astronomical applications for which such a detector could play a major role, either at optical wavelengths from the ground $(\lambda>350 \mathrm{~nm})$, or at ultraviolet and optical wavelengths $(\lambda \sim 100-2000 \mathrm{~nm})$ from space, would be: (a) faint object broad-band spectroscopy which, if coupled to a panoramic imaging capability, would lead to the direct determination of the spectral energy distribution and redshift of every object detected in the field either through the observation of continuum discontinuities (Lyman edge) or through the observation of line centroids; (b) highly efficient spectrophotometry of variable objects, with time resolution well below $1 \mathrm{~ms}$; (c) order separation when coupling a strip of STJs to a high resolution echelle-type spectrograph; (d) speckle imaging, speckle spectroscopy, adaptive optics, interferometric fringe detection, and other atmospheric correction techniques requiring high time resolution.

An STJ array is already being considered as a possible European-contributed camera for Hubble Space Telescope (STECF 1995), and the benefits of the time and energy resolution for a space interferometer have also been considered (Perryman \& Peacock 1995).

Acknowledgements. The authors acknowledge the technical support of A. van Dordrecht (ESTEC), R. Venn of Cambridge Microfab Ltd (UK) and D. Goldie of Oxford Instruments Ltd (UK).

\section{References}

Bardeen J., Cooper L., Schrieffer J.R., 1957, Phys. Rev. 108, 1175

Booth N.E., 1987, Appl. Phys. Lett. 50, 293

Delamere A., 1992, ESA SP-356 111

Dravins D., 1994, ESO Messenger 78, 9

Fano U., 1947, Phys. Rev. 72, 26

Giaever I., 1960, Phys. Rev. Lett. 5, 464

Goldie D.J., Brink P.L., Patel C., Booth N.E., Salmon G.L., 1994, Appl. Phys. Lett. 64, 3169

Gray K., 1978, Appl. Phys. Lett. 32, 392

Josephson B.D., 1962, Phys. Rev. Lett. 1, 251

de Korte P.A.J., 1992, ESA SP-356 41

Kurakado M., 1982, Nucl. Instr. Meth. 196, 275

Lumb D., van Dordrecht A., Peacock A., et al., 1995, SPIE 2518,258

Morohashi S., Imamura T., Hasuo S., 1992, J. Appl. Phys. 72, 7, 2969

Peacock A., Verhoeve P., Rando N., 1996a, Nat (in press)

Peacock A., Verhoeve P., Rando N., 1996b, J. Appl. Phys. (submitted)

Perryman M.A.C., Foden C.L., Peacock A., 1993, Nucl. Instr. Meth. A325, 319

Perryman M.A.C., Peacock A., 1995, "Future Possibilities of Astrometry from Space", ESA SP-379, 207

Petroff M.D., Stapelbroeck M.G., 1989, IEEE Trans Nucl. Sci. 36, 158

Rando N., Peacock A., van Dordrecht A., et al., 1992, Nucl. Instr. Meth. A313, 173

Rando N., Verhoeve P., van Dordrecht A., 1996, Nucl. Instr. Meth. A370, 85

STECF Newsletter No. 23, September 1995

Timothy J., 1988, In "Instrumentation for Ground-Based Optical Astronomy, Present and Future", Robinson L.B., (ed). Springer-Verlag, New York, p. 516

Verhoeve P., Rando N., Verveer J., 1996, Phys. Rev. B. 53, 2, 809

Weast R.C., 1981, CRC Handbook of Chemistry and Physics, CRC Press Inc.

Weaver J.H., Lynch D.W., 1973, Phys. Rev. B 7, 4311

Weaver J.H., Krafka C., Lynch D.W., Koch E.E., 1981, Physik Daten 18-1

Wood G.H., White B., 1969, Appl. Phys. Lett. 15, 237 